\title{
Einleitung
}

\section{Die Handschriften}

\section{Theodorets Kirchengeschichte}

$\mathrm{A}=$ Codex Bodleianus Auct. E II 14 (misc. 42) [B bei Raeder, Graecarum affectionum curatio], perg., s. XI: 167 Blätter, 37 Zeilen auf der Seite; schöne und sehr korrekte Schrift. Inhalt: KG (Bl. 1-79),

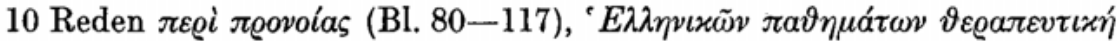
(Bl. 118-167). A ist systematisch durchkorrigiert worden $\left(\mathrm{A}^{\mathrm{c}}\right)$. Die von A abgeleiteten Handschriften sind alle nach Vornahme der Korrektur abgeschrieben. Erwähnenswert ist davon nur:

$\mathrm{E}=$ Codex Basileensis A III 18 [Omont 80], bombyc., s. XIII,

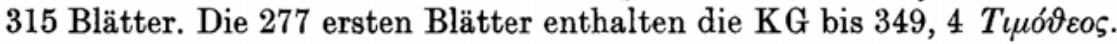
„Die Handschrift ist gebunden; aber alle Blätter, welche die KG enthalten, und zwar nur diese, sind lose. Ich habe so auf rein äußerliche Weise die interessante Feststellung machen können, daß diese Handschrift direkt zum Druck der Baseler editio princeps hingegeben gewesen ist. Man sieht in ihr in regelmäßigen Abständen Zeichen in Rot, die für den Drucker bestimmt sind."

$\mathrm{L}=$ Codex Laurentianus X 18 [L bei Raeder], perg., s. XI; 210 Blätter, 37 Zeilen auf der Seite. Inhalt: ' $E \lambda \lambda$. $\pi$. $\vartheta \varepsilon \varrho \alpha \pi \varepsilon v \tau \iota x \eta ́, ~ K G ~\left(B l . ~ 66^{\mathrm{r}}\right.$

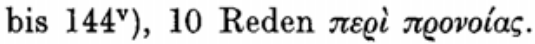

$\mathrm{B}=$ Codex Bodleianus Auct. E 4, 18 (misc. 61), perg., s. X; 144 Blätter, 25 Zeilen auf der Seite; sehr schöne Schrift, aber ziemlich fehlerhafte Orthographie. Die Handschrift, die im Anfang verstümmelt ist (inc. S. 80, 14), hat noch dreimal Blätter verloren, die von jüngeren Händen ersetzt sind:

$\mathrm{B}^{2}\left(\mathrm{Bl} .17^{\mathrm{r}}-24^{\mathrm{v}}=\mathrm{S} .118-137\right), 26$ Zeilen auf der Seite, flüchtige Schrift; perg., s. XII/XIII.

$\mathrm{B}^{3}\left(\mathrm{Bl} .25^{\mathrm{r}}-32^{\mathrm{r}}=\mathrm{S} .137-152\right), 26$ Zeilen auf der Seite, steile und regelmäßige Schrift; perg., s. XII.

$B^{4}\left(B 1.97^{r}-104^{v}=\right.$ S. $\left.282-297\right), 26$ Zeilen auf der Seite; sehr eilige Hand, perg., s. XII/XIII. 
Außer der KG enthält B einen Brief von Proclus an die Bischöfe

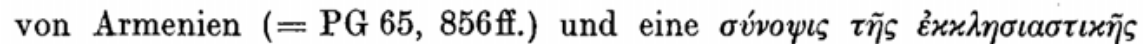

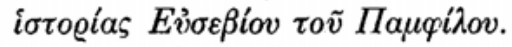

$\mathrm{H}=$ Codex Parisinus 1442, bombyc., s. XIII; 165 Blätter, 27-28

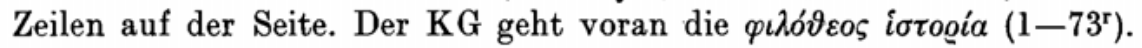
Handschrift stark mitgenommen, Schluß verstümmelt.

$\mathrm{N}=$ Codex 211 der Bibliothek des Klosters Vatopädi auf dem Athos, perg., s. XIII; 153 Blätter, 36 Zeilen im Durchschnitt auf der Seite. Die von Tieren ziemlich beschädigte Handschrift enthält nur die Kirchengeschichte.

$\mathrm{G}=$ Codex Angelicus [Rom] 41, perg., s. XII/XIII, 140 Blätter. Enthält: Theodoreti Quaestiones in Octateuchum und von anderer Hand, $56^{\mathrm{r}}-140^{\mathrm{v}}$, die $\mathrm{KG}$ in schöner, regelmäßiger Schrift, aber mit vielen orthographischen Fehlern. In zwei Spalten geschrieben, 44 Zeilen auf der Spalte.

$\mathrm{S}=$ Codex Scorialensis X III 14, perg., s. XII; 183 Blätter in zwei Spalten, 26 Zeilen auf der Spalte, sehr schön und korrekt geschrieben. Außer der KG (Bl. 1-163 $)$ ist das Leben des St. Anastasius Persa und der Anfang der Predigt Gregors von Nyssa über Theodorus (PG 46, $736 \mathrm{ff}$.) in der Hs. enthalten.

$\mathrm{P}=$ nur in Bruchstücken erhaltener Codex, seinerzeit im Besitz des Professors A. Papadopulos-Kerameus in St. Petersburg, jetzt in der Staatl. Öffentl. Saltykov-Ščedrin-Bibliothek zu Leningrad; perg., Ende des IX. Jahrh., 31 Zeilen auf der Seite. 26 Blätter in kleiner Schrift. Über zwei andere zugehörige Blätter im Codex Parisinus 1248 suppl. gr. s. Revue de Philologie 33 (1909) 81 u. $245 \mathrm{f}$.

$\mathrm{V}=$ Codex Vaticanus 628 , perg., s. XI; 253 Blätter, 21 Zeilen auf der Seite; sehr sorgfältige Schrift. Nur KG. V ${ }^{2}$ (zweite Hand) $113^{\mathrm{r}}-193^{\mathrm{v}}$ $=\mathrm{S} .187,15-276,12$. Aus $\mathrm{V}\left(+\mathrm{V}^{2}\right)$ peinlich genau abgeschrieben: Codex Marcianus 337, perg., s. XV; 370 Blätter, 46 Zeilen auf der Seite. Es ist dies die wohlbekannte Handschrift, die, aus der Bibliothek Bessarions stammend, die Kirchenhistoriker enthält. Theodoret nimmt darin die Blätter $86^{\mathrm{r}}-135^{\mathrm{v}}$ ein. Aus diesem Marcianus ist dann abgeschrieben der Codex Parisinus 1440, chart., s. XVI; 153 Blätter. Die Handschrift stammt aus der Bibliothek des Diplomaten und Humanisten Jean de Pins, Bischofs von Rieux (1470-1537) und wird deshalb häufig als Codex Pini zitiert.

$\mathrm{F}=$ Codex Parisinus 1433 [D bei Schwartz], perg., s. XI/XII, 318 Blätter mit 33 Zeilen auf der Seite; am Anfang und am Schluß verstümmelt. Inhalt: KG des Eusebius, Auszug aus dem Leben Constantins (II 24-42) und Theodorets KG $\left(184^{\mathrm{r}}-\right.$ Schluß). Zahlreiche Risse und Löcher beeinträchtigen den Text. 
$\mathrm{R}=$ Codex Parisinus $1603 \mathrm{~A}$, perg., s. XI; 29 falsch numerierte Blätter mit 25 Zeilen auf der Seite. Schöne Schrift mit Korrekturen von zweiter Hand.

$\mathrm{D}=$ Codex Parisinus 994, chart., s. XV; 175 Blätter, 33 Zeilen auf der Seite; kleine, schwer lesbare Schrift. Inhalt: Christus patiens, Theodorets KG I $\left(120^{r}-146^{r}\right)$, Aesopi fabulae; jedes Werk von andrer Hand geschrieben.

Aus D und $\mathrm{H}$ ist abgeschrieben Codex Berolinensis Phillipps 1619, chart., s. XVI. Enthält die Bücher I (nach D) und III, IV, V (nach H) der $K G$.

2. Handschriften derindirekten und der Parallelüberlieferung

$\mathrm{T}=$ Codex Marcianus 344, perg., s. XIII (Ende); 231 Blätter, 31 Zeilen im Durchschnitt auf der Seite. Inhalt: Tripartita des Theodorus Lector Buch I u. II, Sozomenus V-IX.

$\mathrm{W}=$ Codex Vindobonensis historicus graecus 8, perg., Anfang des XIV. Jahrh.; 498 Blätter, 40 bis 42 eingeritzte Zilen. Es ist die Handschrift der KG des Nicephorus, nach W. Weinberger, Sitzungsberichte der kais. Akad. d. Wiss. in Wien, Philos.-hist. Klasse, Bd. 159, Wien 19008, 6. Abh., S. 61 von dem Verfasser selbst oder von seinem Schreiber geschrieben.

Für Cassiodors lateinische Übersetzung hat Parmentier verglichen:

$\mathrm{L}=$ Codex Leidensis Vossianus lat. fol. 62, perg., s. XI (beste dieser drei Handschriften),

$\mathrm{C}=$ Codex Camaracensis (Cambrai) 685 (625), perg., s. X/XI (nur fürs 1 . Buch benutzt),

$\mathrm{P}=$ Codex Parisinus lat. 5082, perg., s. $\mathrm{X} / \mathrm{XI}$ (nur für die beiden ersten Bücher benutzt).

Für die syrischen Übersetzungen $(=\Sigma)$ sind die wichtigsten Handschriften:

Vaticanus syr. 145, perg., s. VII (s. z. B. S. 131 ff., 167 ff., 303 ff.) und Britisches Museum add. 14, 529, perg., s. VII/VIII (s. S. $297 \mathrm{ffi}$.). Die syrischen Lesarten sind von Prof. M. A. Kugener (Brüssel) festgestellt.

Nicetas Acominatus wurde nach Codex Parisinus 1234, s. XIII kontrolliert.

„Endlich hat für die drei Urkunden des Theodoret (I 9, II 8 und 22), die in der so wertvollen lateinischen Überlieferung des Codex Veronensis 60 (perg., s. VII) erhalten sind, Herr Professor Hans Lietzmann die große Güte gehabt, mir die photographische Wiedergabe zur Verfügung zu stellen, die er von dieser Handschrift besitzt." 


\section{Die Gruppierung der Handschriften}

In den beiden ersten Büchern hatte der Schreiber von A eine im allgemeinen vortreffliche Vorlage. Als er mit der Abschrift des dritten Buches beginnen wollte, stieß er auf eine Handschrift, die ihm einen gelehrteren Eindruck gemacht zu haben scheint. Ihr folgte er von jetzt an. Ja, er (oder ein anderer) verbesserte sogar den Text der beiden schon fertiggestellten Bücher nach dieser Handschrift, die leider zu der minderwertigen zweiten Klasse gehörte und eine Parallelhandschrift von L war. „In der Mehrzahl der Fälle sind nach der Korrektur von $\mathrm{A}^{\mathrm{c}}$ die ursprünglichen Lesarten von A nicht mehr zu lesen, aber sie lassen sich mit absoluter Sicherheit durch Vergleichung mit den besten Handschriften oder mit den Zeugnissen der Parallelüberlieferung wiederherstellen. $\mathrm{Da} \mathrm{A}^{\mathrm{c}}$ sich immer zu einer Handschrift des Zweiges L stellt, kann der Leser tatsächlich im allgemeinen den Schluß ziehen, daß A die entgegengesetzte Lesart hatte". $A^{\mathrm{c}} \mathrm{L}$ und (vom dritten Buche an) AL bilden also eine Sondergruppe (y) der zweiten Klasse.

Der beste Vertreter der ersten Klasse ist B. Leider fehlt B für einen großen Teil des ersten Buches. $\mathrm{B}^{2}$ und $\mathrm{B}^{3}$ (s. S. IX) schreiben eine $\mathrm{L}$ sehr nahestehende Handschrift ab, $\mathrm{B}^{4}$ gibt dagegen eine bessere Handschrift wieder. B war eng verwandt mit der Hs., die Cassiodor benutzte. Gemeinsame Auslassungen und Fehler beweisen, daß HNGSP wenigstens vom zweiten Buch an auf einen gemeinsamen Archetypus $=\mathrm{r}$ zurückgehen, der mit Varianten am Rande versehen war. Innerhalb dieser r-Gruppe stellen HN $(=\mathrm{n})$ und GSP $(=\mathrm{s})$ zwei verschiedene Fassungen dar, von denen s viel fehlerhafter als $\mathrm{n}$ ist. Der an sich treffliche Archetypus der n-Gruppe war offenbar in schlechtem Zustand. Daher lassen $\mathrm{N}$ und besonders $\mathrm{H}$ häufig unter Aussparung leeren Raumes Worte aus. Von der s-Gruppe rangiert am höchsten das Bruchstück $\mathrm{P}$, dann folgt $\mathrm{S}$, am minderwertigsten ist $\mathrm{G}$.

Im ersten Buch gehen $\mathrm{n}$ und $\mathrm{s}$ so weit auseinander, daß sie nicht $\mathrm{zu}$ einer Gruppe zusammengefaßt werden können. Das beruht darauf, daß n sich in diesem Buche mehr von aus der schlechten zweiten Klasse stammenden fehlerhaften Lesarten freigehalten hat als $\mathbf{s}$. So ist im ersten Buch $n$ zuweilen sogar besser als A. Überhaupt war der Archetypus n mit A verwandt, und beide hatten besondere Beziehungen zur Quelle von T, der Tripartita des Theodorus Lector.

Somit stellt sich das Stemma der ersten Klasse folgendermaßen dar. (Die gewundene Linie nom stellt Beeinflussung der ersten Klasse durch die zweite, die unterbrochene einen von der ersten auf die zweite ausgehenden Einfluß dar): 


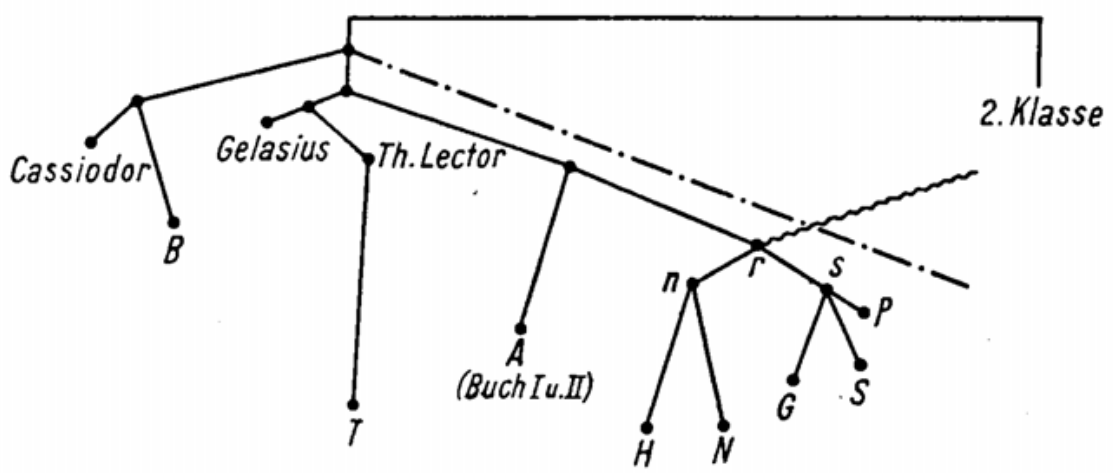

In der zweiten Klasse bilden $\mathrm{DB}^{2} \mathrm{~B}^{3} \mathrm{~L}$, wozu in der Regel FVR treten, die Gruppe z. D steht L sehr nahe, hat aber noch eine andere Hs. zu Rate ziehen können und vermeidet ziemlich häufig Fehler seiner Gruppe. FVR bilden eine besondere Untergruppe $=\mathrm{v}$. Das war an sich die am meisten verderbte Gruppe. Aber S. 187, 15 setzt in V eine zweite Hand ein $\left(\mathrm{V}^{2}\right)$, die von der ersten Klasse beeinflußt ist: $V^{2}$ stellt sich zu B. Auch wo im fünten Buch die erste Hand von V wieder einsetzt, bewahrt die Handschrift im allgemeinen ihren Wert. Aber auch F bietet von S. 249 ab Lesarten, die denen von $\mathrm{V}^{2}$ bzw. V nahestehen. Ähnliches gilt von $\mathrm{B}^{4}$. Das Stemma der zweiten Klasse ist demnach:

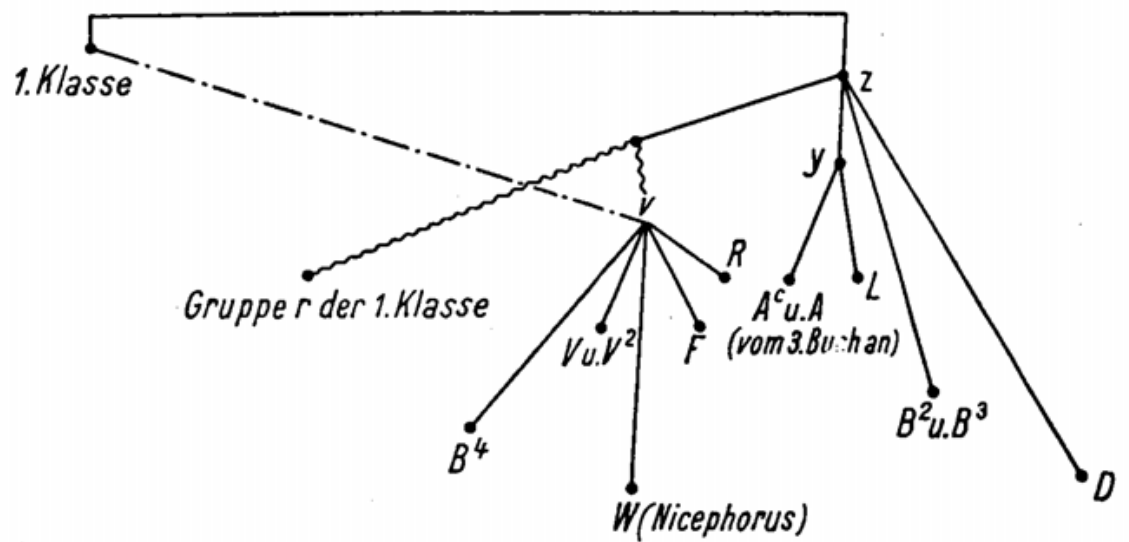

In der Ausgabe sind die Handschriften jedesmal in der ihrem Wert für die Konstituierung des Textes entsprechenden Reihenfolge angeführt. 
Es ist folgende:

Erstes Buch: B A HN (n) GS (s) DL + FV (v) $=z$.

Zweites Buch: B A HN $(\mathrm{n})+\mathrm{GS}(\mathrm{s})=\mathrm{r}, \mathrm{B}^{2}\left(\operatorname{oder} \mathrm{B}^{3}\right) \mathrm{L}+\mathrm{FVR}(\mathrm{v})=\mathrm{z}$.

Drittes Buch bis S. 187: B HN(n) + GS(s) = r AL(y) FVR (v).

Von S. 187 ab: B V ${ }^{2} \mathrm{HN}(\mathrm{n})+\mathrm{GSP}(\mathrm{s}) \doteq \mathrm{r}$ AL(y) F.

Von S. 249 ab: B (oder B $\mathrm{B}^{4} \mathrm{~V}^{2}$ (oder V) F HN(n) $+\mathrm{GSP}(\mathrm{s})=\mathrm{r}$ $\mathrm{AL}(\mathrm{y})$.

\section{Theodorus Lector, Cassiodor, Nicephorus}

Um 530 schuf Theodorus Lector aus den Kirchengeschichten des Socrates, Sozomenus und Theodoret seine Historia tripartita. Hätten wir sie in der ursprünglichen Fassung, so würde sie für die aus Theodoret entnommenen Abschnitte also eine Handschrift des 6. Jahrhunderts darstellen. Wir besitzen aber nur die beiden ersten Bücher dieses vier Bücher umfassenden Werkes in einer Hs. des 13. Jahrhunderts (T). Glücklicherweise hat Cassiodor bis zum siebenten Kapitel des zweiten Buches den Text der Tripartita sklavisch übersetzt (J. Bidez: La traditicn manuscrite de Sozomène et la Tripartite de Théodore le Lecteur, Leipzig 1908 [TU 32, $2^{\text {b}}$ ], $\mathrm{S} .51 \mathrm{ff}$.$) . Vergleicht man \mathrm{T}$ mit dieser Übersetzung, so zeigen gemeinsame Fehler, daß schon im 6. Jahrh. die Tripartita in keinem vollkommenen Zustand war. Bis zum 13. Jahrh. sind zahlreiche weitere Entstellungen hinzugekommen. Theodorus Lector hat sich auch mehrfach erlaubt, den Text Theodorets umzugestalten. In diesen Fällen hat sein Zeugnis natürlich keinen Wert. Es ist dann im Apparat mit T* bezeichnet.

Von II 7 an benutzt Cassiodor eine eigene Theodorethandschrift und stellt dann immer eine bessere Überlieferung als T dar. „Ich habe den Eindruck, daß, gerade umgekehrt wie Theodorus Lector, Cassiodor niemals, wenigstens soweit es Theodoret betrifft, den Text seiner griechischen Handschrift mit Vorbedacht umgearbeitet oder gefälscht hat." Seine Genauigkeit im Übersetzen ist von großer Bedeutung. „Die besten Hand-

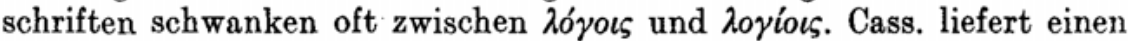

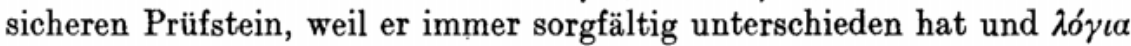
mit eloquia übersetzt. Die gleiche Sorgfalt verwendet er auf die treue Wiedergabe der Tempora beim Verb, der Partikeln und der Wortstellung. Endlich sind selbst die Auslassungen von Cass. lehrreich, weil sie oft bezeugen, daß er schon einen unübersetzbaren Text vorfand $(106,23$. $257,17 / 18.272,4 / 5)$ “.

Nicephorus Xanthopulus (14. Jh., vgl. K. Krumbacher, Geschichte der byzantinischen Litteratur, 2. Aufl., München 1897, S. $291 \mathrm{ff.}$.) verflicht in seiner KG meistens die Version Theodorets mit der des Socrates und Sozomenus. Von Wert ist sein Text $(=$ W, s. S. XI) natürlich nur 
da, wo er den Theodoret allein benutzt hat, also in den nur bei diesem überlieferten Urkunden und z. B. in dem Kapitel über die Märtyrer Persiens. Hier hat gerade seine Handschrift in Übereinstimmung mit dem vortrefflichen Cod. L des Cassiodor es Parmentier 345, 14 erlaubt, die

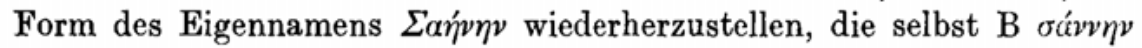
geschrieben hatte. Die Handschrift, die Nicephorus benutzte, zeigt eine gewisse Verwandtschaft mit $\mathrm{F}$ und war wohl mit Varianten am Rande versehen, weshalb bei ihm Lesarten verschiedener Klassen auftreten.

\section{Die Textgeschichte}

„Theodoret schreibt mit der Emphase eines Predigers unablässig cum ira gegen die Ketzer, cum studio hinsichtlich der Rechtgläubigen; er wird nicht müde, Geschichten zu sammeln und zu stilisieren, in denen die priesterliche Anmaßung gegenüber der Staatsgewalt triumphiert. Durch diesen Charakterzug mußte er weit mehr als Socrates und Sozomenus, die doch noch Laien waren, die ganze Freude der Priester und Mönche ausmachen; nicht minder zog er die Theologen durch seine reiche Urkundensammlung an. Darum ist er auch unter den Kirchenhistorikern der am meisten gelesene und abgeschriebene gewesen ... Frühzeitig bedeckten sich die Ränder vieler Exemplare mit Anmerkungen, und die meisten Varianten, die den minderwertigen Handschriften gemeinsam sind, reichen jedenfalls sehr weit zurück. Im allgemeinen sind die durchkorrigierten Handschriften wie L, F und wie $\mathrm{V}$ in dem ersten Teil nicht weniger alt als die anderen. ... Der Zustand des Textes, der für die Archetypen n, s, v usw. vorauszusetzen ist, geht sonach auf alte Rezensionen zurück. Diese rühren ohne Zweifel von einer ähnlichen Bearbeitung her, wie sie auch andere Kirchenhistoriker betroffen hat und wie sie z. B. bei Euagrius neben der guten Überlieferung A eine revidierte Rezension $\mathrm{z}$ hat entstehen lassen. ... Aber diese verschiedenen Rezensionen, die seit dem IX. Jahrhundert und noch früher existierten, nahmen ihrerseits auch wieder Varianten ältern Datums auf. Solche Varianten sind z. B. die, deren Alter durch die Übereinstimmung von Cass. oder selbst T mit einer Handschriftenklasse gestützt wird." Interessant ist besonders der im Glaubensbekenntnis des Damasus (S. 300,4) interpolierte Anathematismus Cyrills, der natürlich in eine Zeit zurückgeht, wo der nestorianische Streit noch aktuell war. Antinestorianisches auch S. 224, 1f. im Apparat. Daneben zeigt die Textgeschichte auch ,die im allgemeinen so häufige Tendenz, in alle Handschriften mehr und mehr die lectio facilior oder einfach die Korrekturen einer neuen Rezension einzuführen. Der Fall des Codex $\mathrm{A}$, wo $\mathrm{A}^{\mathrm{c}}$ überall die richtigen Les- 
arten von A unterdrückt, ist dafür das charakteristischste Beispiel". Übrigens ist auch in B an einer Stelle (S. 307f. Apparat) eine lange Interpolation eingeschoben worden.

„A As unsrer ganzen Untersuchung ergibt sich, daß die Überlieferung im allgemeinen erlaubt, den Text des Theodoret auf Grundlagen von bemerkenswerter Sicherheit aufzubauen. Mit Handschriften wie A, n, $\mathrm{B}, \mathrm{V}^{2}$, bei besonnener Verwertung der syrischen Bruchstücke, der Tripartita und Cassiodors - ohne jetzt von den so zahlreichen Anleihen in den byzantinischen Autoren zu sprechen - hat man fast immer das Mittel, die Überlieferung zu kontrollieren und zu unterscheiden, was selbst in den besten Handschriften Fehler oder Entstellung ist."

„Unter diesen Umständen konnte nicht die Rede davon sein, daß zu dem bequemen System gegriffen würde, nur einer Überlieferung, wäre es auch die von B, mechanisch zu folgen. B allein z. B. gegen An T Cass. hat kaum Aussicht, das Richtige zu bieten; Lesarten von A oder An lassen sich als vortrefflich erweisen. Ebenso ist es mit Lesarten von $n$ oder $\mathrm{r}$, während solche von $\mathrm{zv}$ fast immer dem Verdacht unterliegen, falsch zu sein, wie es der Stammbaum der Handschriften zeigt. Es mußte also in jedem Fall eine kritische Wahl unter den Zeugnissen getroffen werden, deren Zahl, Wert und Gruppierung fortwährend wechseln, und diese Wahl hat im allgemeinen große Aussicht, uns dem Urtext Theodorets nahe zu bringen."

\section{Der Prosarhythmus in der KG}

Nach dem Meyerschen Klauselgesetz sollen zwischen den beiden letzten akzentuierten Silben am Satzschluß mindestens zwei unbetonte Silben stehen. Auch ein Vielfaches von zwei, also vier oder sechs Silben (mit Nebenton), ist beliebt, während eine ungerade Zahl von Zwischensilben, also 1, 3, 5, 7 tunlichst gemieden wird. Parmentier hat diesem Gesetz, obwohl Wilhelm Meyer es bereits 1891 bekannt gemacht hatte, keine Beachtung geschenkt. Darauf hingewiesen zu haben ist das Verdienst von Wilh. Göber in seiner halleschen Dissertation von 1924 „Quaestiones rhythmicae imprimis ad Theodoreti historiam ecclesiasticam pertinentes" (als Buch Berlin 1926). Es fragt sich nur, ob durch diese Nichtbeachtung großer Schade entstanden ist. Zunächst wird man freilich fragen müssen, ob Theodoret das Gesetz tatsächlich beobachtet hat. Diese Frage muß angesichts der von Göber festgestellten Zahlenverhältnisse bejaht werden. Er findet nämlich in der KG 31mal keine Zwischensilbe, 190mal eine, $331 \mathrm{mal} \mathrm{3,176mal} 5$ und 10mal 7 Zwischensilben. Ganz andere Zahlen gibt es dagegen bei geradzahligen $Z$ wischensilben: $3883 \mathrm{mal}$ 
2, 1956mal 4, 80mal 6 Zwischensilben. Aber die Zahl der Ausnahmen ist doch so groß, daß man zum mindesten sagen muß: Theodoret hat in der KG, die ja überhaupt etwas eilig gearbeitet ist, das Gesetz nicht konsequent beobachtet. Göber versucht allerdings auf alle mögliche Weise die Zahl der Ausnahmen herabzudrücken: er mißt $\varepsilon \omega, \underline{\varepsilon}, \alpha a$, faßt $\alpha a i$ und vokalisch auslautende Artikelformen mit folgendem vokalischem Anlaut

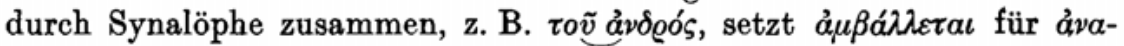

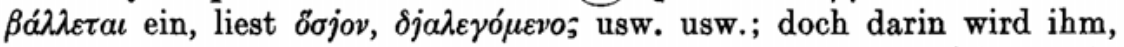
da er je nach Bedarf auch wieder die natürliche Praxis befolgt, wohl niemand beipflichten. Nach dem Urteil von P. Maas (Byz. Z. 26 [1926] 440) sind überdies derartige Messungen der byzantinischen Kunstprosa völlig fremd. Für die Textkritik ergibt sich daraus, daß das Klauselgesetz an sich niemals von entscheidender Bedeutung sein kann, daß es also z. B. verkehrt wäre, lediglich deshalb der Lesart einer sonst minderwertigen Handschrift oder Handschriftengruppe den Vorzug zu geben, weil sie das Klauselgesetz beobachtet. Es ist außerdem nicht so, daß Parmentiers Bewertung der Handschriften durch die Klauselstatistik bestätigt würde, wie Göber S. 71 z. B. von B behauptet (qua ratione feliciter Parmentarii iudicium comprobatur); im Gegenteil! L z. B. hat neben 671 rhythmischen, d. h. klauselgerechten Schlüssen 168 unrhythmische, d.h. ein Viertel, B dagegen 194 unrhythmische neben 568 rhythmischen, d. h. mehr als ein Drittel; das Verhältnis gestaltet sich noch ungünstiger, wenn wir lediglich $\mathrm{B}$ und $\mathrm{B}^{4}$ in Betracht ziehen (188:527), während es bei den minderwertigen Teilen $\mathrm{B}^{2}$ und $\mathrm{B}^{3}$ mit 6:41 recht günstig ist. Das spricht doch dafür, $\mathrm{da} \beta$ gerade von den Schreibern der zweiten Klasse an der Verbesserung der Klauseln gearbeitet worden ist; Göbers Behauptung, daß in allen Handschriften das Verhältnis zwischen rhythmischen und unrhythmischen Schlüssen konstant sei (S. 70, 1), stimmt eben nicht. Trotzdem sind unter den Textverbesserungsvorschlägen Göbers (§13, S. 35-46) manche annehmbar oder wenigstens erwägenswert. Ich werde sie in den Anmerkungen zu den einzelnen Stellen anführen.

\section{Die Urkunden und ihre Uberlieferung}

Der Hauptwert der KG Theodorets beruht auf der Fülle der in ihr mitgeteilten Urkunden, Urkunden zum Teil, bei denen wir über keine Parallelüberlieferung verfügen. Aber gerade diese Urkunden sind vielfach in einem jämmerlichen Zustand. „Dieselben Handschriften, die uns bei den Erzählungen in den Stand setzen, einen korrekten Text aufzustellen, führen uns häufig, wenn es sich um Urkunden handelt, nur zu einem 
fehlerhaften und unerklärlichen Text. In manchen Fällen stand ein Fehler, selbst wenn er schon in der ersten Abschrift des Theodoret vorlag, nicht in dem Text, der kopiert werden sollte. So haben z. B. 126, 13 die Worte $\varepsilon \dot{v} \vartheta \dot{v} \varsigma \tau \varepsilon \mu \dot{v} v$, die durch Athanasius und Socrates gesichert, von allen Handschriften und von der Tripartita ausgelassen werden, eine Spur ihres Vorhandenseins in der Vorlage des Theodoret selbst in den von den besten

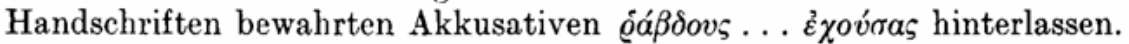
Aber im allgemeinen gehen die Fehler der Urkunden auf Theodorets eigene Quelle zurück und standen bereits in den Aktenstücken, die er seinem Schreiber zum Kopieren gab. ... Es läßt sich also behaupten, daß Theodoret schon in seinen Sammlungen Urkunden in schlechtem Zustand fand und daß mehrere derselben von Anfang an in ziemlich elender Weise aus dem Lateinischen übersetzt waren, besonders II 22; IV 8 und 9." Und niemals ist in der Überlieferungsgeschichte der KG Theodorets versucht worden, Fehler in den Urkunden durch Heranziehung der Parallelïberlieferung zu verbessern. Von Theodoret bis Nicephorus können wir eine bestimmte Arbeitsmethode feststellen: „Die Urkunden wurden einfach nach einem dem Schreiber bezeichneten Text zum Kopieren gegeben, und der Autor nahm sich allem Anschein nach oft nicht die Mühe, die Abschrift desselben wieder durchzusehen. ... Daß Theodorus Lector, der dauernd die drei Synoptiker zur Hand hat, jemals in den Urkunden ihre Texte ineinander gearbeitet hätte, davon habe ich kein sicheres Anzeichen entdeckt. Cassiodor hält bei seinen Übersetzungen von Aktenstücken treu daran fest, die Rezension des von ihm gewählten Autors lateinisch wiederzugeben. Wenn diese Rezension verderbt oder unverständlich ist, verliert er den. Faden, setzt neu an oder gibt auch alle Hoffnung auf und läßt das Unverständliche einfach aus. Niemals kommt er auf den Gedanken, daß z. B. Socrates, dem er den unmittelbar folgenden Abschnitt entnimmt, ihm den Schlüssel zu der Stelle geben könnte, die ihm im Augenblick bei Theodoret Not macht. Noch viel weniger läßt er sich bei den aus dem Lateinischen ins Griechische übertragenen Urkunden auch nur einfallen, was in der Gegenwart jeder tun würde, sich der mühsamen Arbeit einer Rückübersetzung zu entziehen und den Text des lateinischen Originals zu ermitteln, das wieder aufzufinden ihm zuweilen wohl ein leichtes gewesen wäre." Gelasius von Cyzicus freilich ,hat die Urkunden nicht einfach kopieren lassen, sondern sie diktiert oder selbst geschrieben, indem er sie öfters interpolierte, fälschte und überarbeitete. Aber diese Änderungen entspringen seiner freien Erfindung, ohne eine Spur von Mischung mehrerer Quellen".

Theodoret I 9, wo die anderwärtige Überlieferung sehr reich ist, ,,ist die theodoretische Version besonders fehlerhaft“. Aber „,kein Schreiber 
hat daran gedacht, auf Socrates zurückzugreifen, um sie zu verbessern und offenbare Auslassungen wie z. B. das Wort $\tau \mu \eta^{\prime} v 40,7$ zu ergänzen“"

Unter diesen Umständen hat der Herausgeber der KG Theodorets natürlich zunächst die Aufgabe, den Originaltext Theodorets wiederherzustellen, soweit das möglich ist. Er wird aber darüber hinaus auch die Entstellungen, die dieser Originaltext bereits aufwies, feststellen und verbessern müssen, damit die Urkunden selbst verständlich werden.

\section{Die Abschreiber}

„Das Verzeichnis der Abschreiber, das sich S. 384f. dieser Ausgabe findet, beabsichtigt keineswegs alle Autoren aufzuzählen, die aus der KG Stellen entlehnt haben. Ich habe mir besonders angelegen sein lassen, die Autoren heranzuziehen, für welche zuverlässige kritische Ausgaben vorliegen, z. B. Georgius Monachus und Theophanes von de Boor sowie das Synaxarium ecclesiae Constantinopolitanae von Delehaye."

„Die Chronik des Michael Syrus (Buch 7 u. 8) hat uns einen Abriß von einem sehr großen Teil von Theodorets Werk erhalten. Aber diese summarische Übersicht kann nicht die Dienste leisten, die man sonst von so getreuen Abklatschen erwartet, wie es die syrischen Übersetzungen sind, und die Fälle, wo ihr Zeugnis mir auch nur ein wenig Nutzen gebracht hat, sind ziemlich selten."

Mit größter Vorsicht ist Gelasius Cyzicenus zu benutzen. „Geradezu gefährlich macht ihn der Umstand, daß er sich vornehmlich darin gefällt, seine Fälschungen in die Urkunden einzuführen, die doch unantastbar sein sollten. ... Er schreibt III 15, 21/22 und 16 Theodoret I 26 von

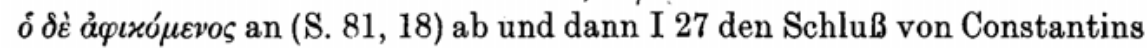
Brief an die Gemeinde von Alexandrien. Aber bevor Gelasius diesen Briefschluß kopiert, verläßt er einen Augenblick seine Quelle, um aus eigner Erfindung sich etwa in dem Sinne vernehmen zu lassen: ,Möge mir keiner der Leser vorwerfen, da $B$ ich den Brief nicht ganz gebe. Ich habe vergeblich alle durchforscht, die über diese Ereignisse geschrieben haben, ich habe ihn nieht vollständig wiedergegeben gefunden. ... Ich bin darüber noch trostloser als ihr alle. So also lautete der Schluß des Briefes.' Gelasius hat sehr schlecht gesucht. Der Brief findet sich vollständig bei Athanasius, Apologia contra Arianos 62, in einem Werk, das er sonach nicht durchforscht hat. Unter den Kirchenhistorikern enthalten weder Socrates noch Sozomenus jenen Schluß des Briefes. Dasselbe Bruchstück findet sich nur bei Theodoret I 27. Unzweifelhaft hat also Gelasius von diesem abgeschrieben. Die Texte des Theodoret und Athanasius decken sich fast genau. Man betrachte, was Gelasius daraus ge- 
macht hat. Ich übergehe einige willkürliche Überarbeitungen im Anfang, lege aber zur Vergleichung die folgenden Stellen vor:

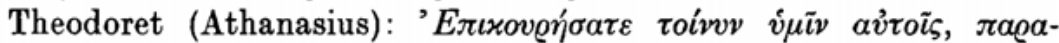

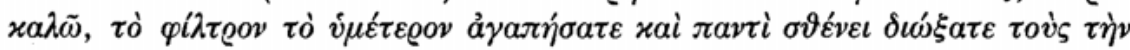

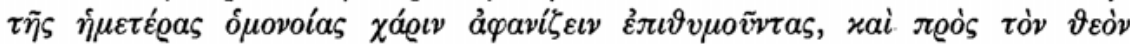

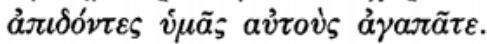

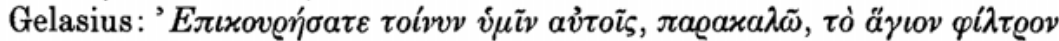

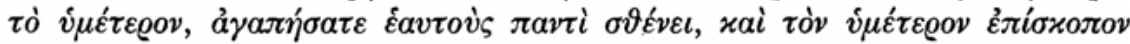

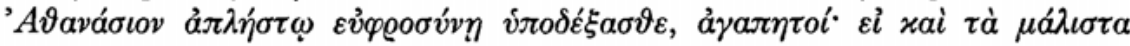

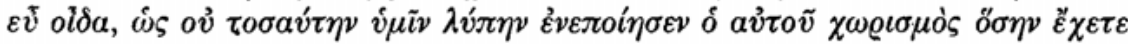

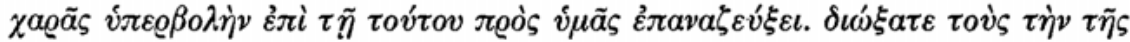

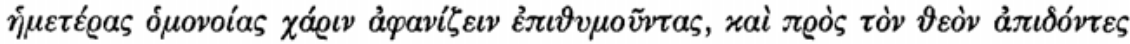

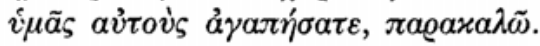

Theodoret hat sieben oder acht Zeilen wiederzugeben unterlassen, die sich noch am Schluß des Briefes bei Athanasius finden. Gelasius fügt,

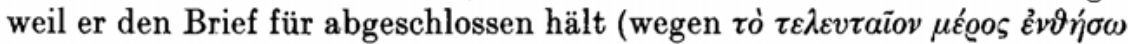

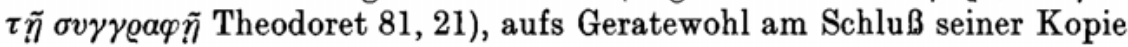

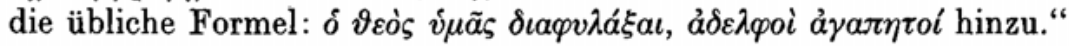

„Bei den Fälschungen der Urkunden scheint unter andern ein Zweck des Gelasius zu sein, die Meinung zu erwecken, daß er aus wertvollen, vor ihm unbekannten Quellen schöpfe."

„In den Erzählungen gefällt sich Gelasius zuweilen ebenfalls darin, Lügen eigener Erfindung einzuführen. So erzählt er III 16 die Affäre der Synode von Antiochien und die Umtriebe gegen Eustathius ausschließlich nach Theodoret I 21. Dennoch behauptet er, daß er ältere Quellen besitze und die Ungenauigkeit seines Vorgängers verbessern werde, all das aus dem lächerlichen Interesse, zu beweisen, da $\beta$ Eusebius von Caesarea in der Angelegenheit von Antiochien keine Rolle gespielt habe."

„Diese wenigen Hinweise genügen, um die Vorsicht zu begründen, die bei der Benutzung des Gelasius für den Aufbau von Theodorets Text anzuwenden ist."

\section{Ausgaben und Ubersetzungen}

1523 Autores historiae ecclesiasticae von Beatus Rhenanus, enthaltend Rufins Übersetzung der KG des Eusebius, die Fortsetzung Rufins (Buch X u. XI) und Cassiodors Tripartita. Hinter dem lateinischen Text der von Cassiodor aus Theodoret übersetzten Urkunden ist der griechische nach $\mathbf{E}$ (S. IX) eingeschoben.

1535 (Basel) erste vollständige Ausgabe der $\mathrm{KG}$ von Hieronymus Frobenius und Nicolaus Episcopius, ebenfalls nach E. In $\mathbf{E}$ hatte eine 
zweite Hand $\left(\mathbf{E}^{\mathrm{c}}\right)$ Lesarten von $\mathrm{B}$ eingesetzt, die in der Ausgabe übernommen wurden. Daneben eigene Korrekturen der Herausgeber. So 240, 7 das zugefügte $\mu \eta^{\prime}$, das alle Ausgaben bis auf Parmentier beibehielten.

1536 (Basel) lateinische Übersetzung des Joachim Camerarius.

1544 (Paris) zweite Ausgabe der KG von Robert Estienne (Stephanus). Grundlage die Baseler editio princeps. Daneben H (S. X) benutzt und sehr vereinzelt der Parisinus 1440 (Codex Pini: S. X).

1570 (Köln) lateinische Übersetzung der griechischen Kirchenhistoriker von Johannes Christophorson, kathol. Bischof von Chichester $(\dagger 1558)$.

1612 (Genf) griechisch-lateinische Ausgabe der Historiae ecclesiasticae scriptores Graeci. Bei Theodoret am Rande die Varianten Christophorsons, die im allgemeinen aus dem Marcianus 337 (S. X) herrühren, bei den Urkunden auch aus der Parallelüberlieferung; vereinzelt handelt es sich auch um Konjekturen Christophorsons.

1642 (Paris) erste Gesamtausgabe Theodorets vom Jesuiten Jacques Sirmond. Die KG fußt auf $\mathrm{H}$; im ersten Buch eine Reihe Lesarten von dem aus D abgeschriebenen Berolinensis (S. XI). Hier zum ersten Male das Verzeichnis der Bischöfe am Schluß des fünften Buches nach B.

1673 (Paris) Theodorets KG mit neuer lateinischer Übersetzung und erläuternden Anmerkungen von Henri de Valois. An neuen Hilfsmitteln hatte Valois eine Kollation von $\mathbf{A}$, in der aber $\mathbf{A}$ und $\mathbf{A}^{\mathrm{c}}$ nicht unterschieden waren, und für die beiden ersten Bücher eine allerdings recht unvollständige Zusammenstellung von Varianten der griechischen Tripartita (T).

1720 (Cambridge) Neudruck der Ausgabe von Valois durch Reading. 1771 (Halle) dritter Band der neuen Gesamtausgabe Theodorets von J. L. Schulze mit der von Joh. Aug. Noesselt bearbeiteten KG auf S. 722 ff. Der kritische Apparat trägt nur die Varianten der früheren Ausgaben zusammen. Noesselts Ausgabe ist von Migne PG 82 übernommen.

1854 (Oxford) KG von Gaisford. Dieser konnte A und B an Ort und Stelle ausbeuten, hat aber weder die Wichtigkeit einer Unterscheidung zwischen $\mathrm{A}$ und $\mathrm{A}^{\mathrm{c}}$ gesehen noch den Wert von $\mathrm{B}$ hinreichend erkannt. Von Gaisford stammt die von Parmentier beibehaltene Paragrapheneinteilung. Ebenso hat Parmentier die in allen früheren Ausgaben mit Ausnahme der von Sirmond und Noesselt sich findende Zählung der Kapitel nicht geändert und da, wo diese Zählung mit den jedem Buche vorangestellten Kephalaia, die wahrscheinlich auf den Autor selbst zurückgehen, nicht übereinstimmt, dies am Rande vermerkt. Seine Ausgabe erschien 1911 in Leipzig. Voran ging eine 1878 in Kempten er- 
schienene deutsche Übersetzung von L. Küpper; 1926 kam dann eine neue deutsche Übersetzung von Andreas Seider in München heraus (Bibliothek der Kirchenväter, Bd. 51).

\section{Die Quellen}

Als Socrates anfing Kirchengeschichte zu schreiben, da übernahm er einzelne Abschnitte nahezu wörtlich aus der KG des Gelasius von Caesarea (vgl. meinen Aufsatz „Die KG des Gelasios von Kaisareia“, Byz. Z. 46 [1953] 277-301). Bei Theodoret gibt es dergleichen nicht; er ist sogar, man möchte sagen, ängstlich bemüht, auch inhaltlich in dem, was er erzählt, einzelnes immer wieder anders zu gestalten als seine Vorgänger. Nehmen wir die Bekehrung der Iberer! Sie wird außer bei Theodoret I 24 erzählt bei Rufin, der Gelasius von Caesarea übersetzt, X 11, bei Socrates I 20, Sozomenus II 7 und dem ebenfalls seinen Namensvetter mehr oder minder genau ausschreibenden Gelasius von Cyzicus III 10. Bei den Iberern wohnt eine kriegsgefangene fromme Christin. Nun haben die Iberer die Sitte, wenn ein kleines Kind bei ihnen erkrankt, es rund zu tragen, um zu sehen, ob jemand ihm helfen kann. So die übrigen. Bei Theodoret aber besteht die Sitte darin, daß die erwachsenen Iberer einander aufsuchen, wenn sie krank werden, um jene zu befragen, die einmal an der gleichen Krankheit gelitten haben und geheilt worden sind. Aber geheilt wird von der Christin dann doch ein krankes Kind, so daß man die Änderung Theodorets nur als ungeschickt bezeichnen kann. Die Königin hört von der Heilung, und da sie selbst an einer schweren Krankheit daniederliegt, läßt sie die Christin zu sich bitten. Diese weigert sich zu kommen. Da läßt sich die Königin zu ihr tragen. So wieder die übrigen Berichterstatter. Bei Theodoret aber av̉ì่ $\pi \varrho \grave{s}$

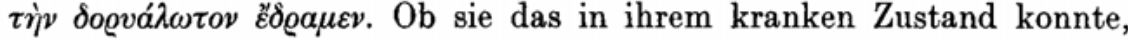
danach fragt Theodoret nicht; er muß nur um jeden Preis anders erzählen als seine Vorgänger. Nach der Heilung will die Königin die Christin reich beschenken. Auch das ist ein neuer Zug bei Theodoret, denn die anderen wissen nichts davon, aber diesmal hat Theodoret wenigstens geschickt geändert. Die Königin ist nun bekehrt, aber ihr Gemahl noch nicht. Dessen Bekehrung wird dann durch eine Sonnenfinsternis herbeigeführt, die ihn am hellen Tage bei der Jagd plötzlich überfällt. Auch hier hat Theodoret wieder seine Besonderheit. Er steigert das Wunderbare des Vorgangs, indem bei ihm sich nur für den König der Himmel verfinstert, während seine Begleiter strahlendes Sonnenlicht haben. Aber hier können wir feststellen, wie Theodoret zu seiner Änderung gekommen ist. Er las

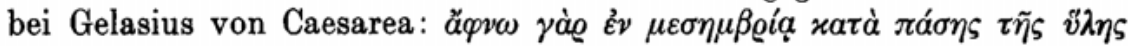




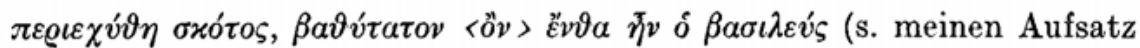
S. 282f.). Diesen Umstand, daß die Finsternis um den König herum am dicksten war, hat er steigern wollen. Damit dürfte erwiesen sein, daß Theodoret den Gelasius von Caesarea benutzt hat. Für Benutzung des Socrates spricht nichts, auch nicht der Umstand, den Parmentier S. LXXXVI anführt, daß Theodoret, wo er den König in seiner Bedrängnis den Gott der Kriegsgefangenen anrufen läßt, diese auf einmal

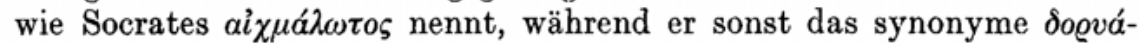

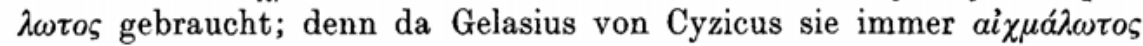
nennt, haben wir Grund, dasselbe von Gelasius von Caesarea anzunehmen. Für Benutzung des Sozomenus, der mehrfach kürzt, spricht erst recht nichts. Parmentier hat vollkommen recht, wenn er annimmt, Theodoret habe Socrates und Sozomenus wohl gekannt, aber sich nicht veranlaßt gefühlt, die Werke dieser Laien zu benutzen, sondern eher zu ihren Quellen gegriffen. Als eine dieser Quellen nennt er den griechischen Rufin. Nun, dieser griechische Rufin ist eben Gelasius von Caesarea, und die KG dieses Gelasius war ein viel umfangreicheres und reichhaltigeres Werk als Rufin (vgl. meinen Aufsatz S. 288ff.), und da feststeht, daß Sozomenus, wenn Socrates von Gelasius abwich, gern wieder zu ihm zurücklenkte, so sprechen auch Übereinstimmungen zwischen Theodoret und Sozomenus, wie Parmentier sie mehrfach anführt, für Gelasius als gemeinsame Quelle beider. Natürlich nicht immer. Sozomenus sowohl wie Theodoret hatten Werke zur Verfügung, die wir nicht mehr haben, deren Vorhandensein wir freilich zuweilen erschließen können. So folgen Theodoret und Philostorgius, wahrscheinlich auch Sozomenus für gewisse Vorkommnisse der Regierung Julians (Überführung der Überreste des Märtyrers Babylas, die Marter des Bekenners Theodorus, den Brand des Tempels von Daphne usw., Theodoret III $10 \mathrm{ff}$.) einem arianischen Autor, auf dessen Benutzung die in der Philostorgiusausgabe von Bidez (Leipzig 1913) S. 96f. durch steile griechische Buchstaben kenntlich gemachten wörtlichen Übereinstimmungen zwischen Theodoret und Philostorgius zurückzuführen sind. Aber es handelt sich dabei nur um einzelne Ausdrücke, nie um ganze Sätze. Wenn also Theodoret I 15 u. 16 zwei Briefe Constantins wiedergibt, die Socrates I 9, $47 \mathrm{ff}$. in der gleichen Reihenfolge und mit zwei wörtlich übereinstimmenden Überleitungssätzen (Theod. 59, 20-24 = Socr. I 9, 50) bringt, so ist das ein ausreichender Beweis dafür, daß Theodoret nicht dem Socrates gefolgt ist, sondern seinem Schreiber dieselbe Urkundensammlung zum Kopieren gegeben hat, die auch Socrates benutzt hat. Die wörtlich übernommenen Sätze gehen zu Lasten des Kopisten. Ebenso hat Theodoret, wie Parmentier nachweist, auch keine Urkunden aus der Vita Constantini des Pseudo-Eusebius ent- 
nommen. Das beweisen die vielen abweichenden Lesarten, vgl. den Apparat 42,$18 ; 43,1.18 ; 45,20 ; 78,13.21 ; 83,15 ; 84,8$. Außerdem erwähnt z. B. Theodoret 46, $20 \mathrm{ff}$. einen Brief Constantins an die Statthalter betreffs Getreidelieferungen zugunsten der Kirchen, von dem in der Vita nichts verlautet, während umgekehrt auf einen von der Vita II 48ff. im Wortlaut gebrachten Brief Constantins bei Theodoret 58, $17 \mathrm{ff}$. bloß hingewiesen wird. Nicht ganz so klar liegt die Sache bei Athanasius, obwohl auch hier mehr für Nichtbenutzung spricht als dagegen. Für das wichtige Schreiben der Synode von Serdica z. B. (II 8) kommt Athanasius schon deshalb nicht als Quelle in Betracht, weil er den wertvollsten Abschnitt dieses Schreibens, das Glaubensbekenntnis, ausläßt. Und in den Urkunden II 20/21 bietet Theodoret einen sowohl von Athanasius (De synodis 55 u. 30) wie Socrates (II 37 u. 41) stark abweichenden Text. So spricht die größere Wahrscheinlichkeit dafür, daß Theodoret lediglich die damals vorhandenen Sammlungen von Synodalurkunden und Brief-

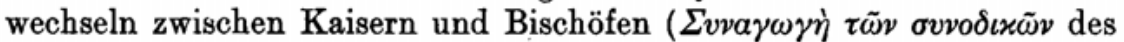
macedonianischen Bischofs Sabinus, Historia acephala Arianorum, Briefsammlungen des Arius und seines Gegners Alexander usw.) benutzt hat. Vgl. dazu P. Batiffol, Byz. Z. 7 (1898) 265ff., 10 (1901) 128ff., Ed. Schwartz, NGG 1904, S. $357 \mathrm{ff}$.

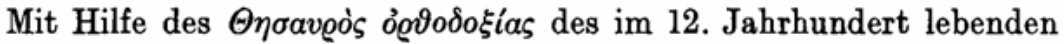
Nicetas Acominatus weist Parmentier eine weitere Quelle Theodorets nach. Es ist der von diesem hochverehrte Theodorus von Mopsuestia. Man vgl. Theodoret 154, 15ff. mit Nicetas V 30 Ende (Vatic. $680 \mathrm{Bl}$. 130 ${ }^{\mathbf{v}}$; Paris.

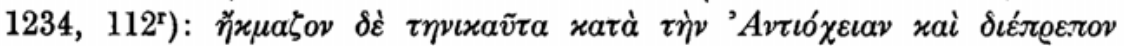

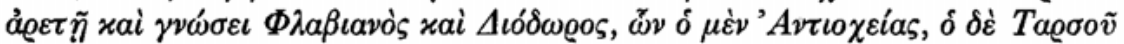

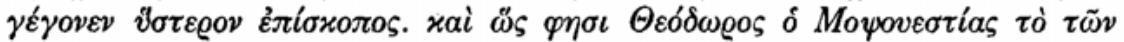

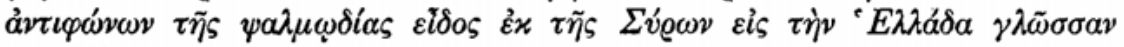

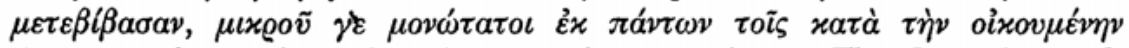

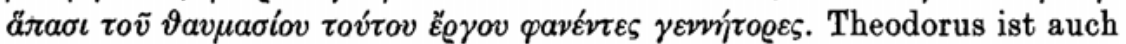
im Thes. V 31 die Quelle des Nicetas; darin findet sich der Satz: $\tau \dot{\alpha} \mu \varepsilon ́ v$

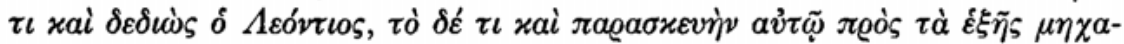

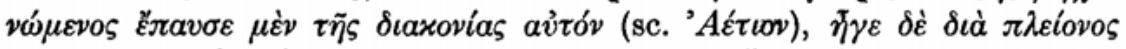
$\tau \iota \mu \tilde{\eta} \varsigma$, der sich inhaltlich mit Theodoret 154, $9 \mathrm{ff}$. deckt. Weiter ist der Abschnitt über die Doxologie bei Theodoret 153, $8 \mathrm{ff}$. und bei Nicetas (V 30 Ende) dem Theodorus entlehnt. Nicetas schreibt: vai $\mu \dot{\nu} v$ oi $\mu \varepsilon \dot{\nu}$

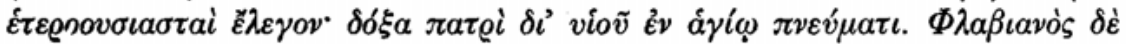

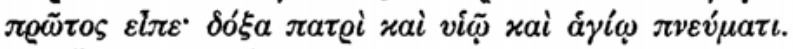

Theodorus scheint gegen den vorher erwähnten arianischen (homöischen) Schriftsteller, den Philostorgius, Sozomenus und zum Teil auch Theodoret benutzen, polemisiert zu haben. Der Unterschied in der Beurteilung des 
Leontius z. B. springt ins Auge, wenn wir Theodoret $153 \mathrm{ff}$. mit Sozomenus

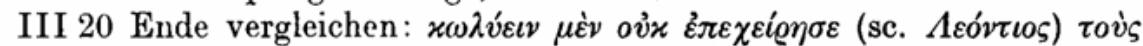

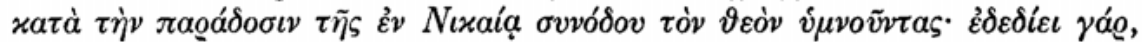

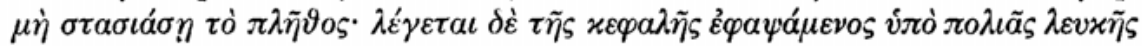

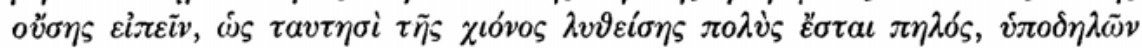

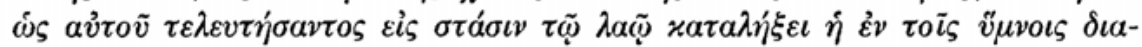

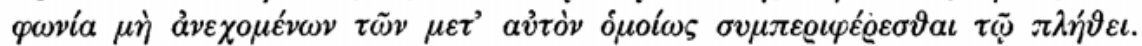
Noch günstiger ist das Urteil des auf dem gleichen Arianer basierenden Chronicon Paschale bei Bidez, Philostorgius S. 215.

Auch I 21 (Absetzung des Eustathius) und II 29 (Eunomius) hat Theodoret nach Parmentiers Meinung den Theodorus benutzt. I 30 dagegen (Verleumdungen gegen Athanasius) spricht die Übereinstimmung zwischen Theodoret und Rufin für Gelasius von Caesarea als Quelle.

„Für die Geschichte der Kirchen im Orient", schreibt Parmentier weiter S. XCV, „lebte Theodoret im Brennpunkt der Nachrichten; er brauchte bloß aus der Literatur des Landes und oft auch aus seinen persönlichen Erinnerungen zu schöpfen, um Aufschlüsse zu geben, die bei den anderen Kirchenhistorikern fehlen". Man vergleiche S. 229 App. den Hinweis auf Schriften der Bischöfe Letoius von Melitene und Amphilochius von Iconium gegen die Messalianer, ferner S. 237, 17 (Briefe des Eusebius von Samosata) sowie $320,10 \mathrm{ff}$. Anderes verdankt er mündlicher Berichterstattung: 191, 25; Religiosa historia 2, wonach er die Geschichte von der Sendung des Acacius an den Eremiten Julianus Sabas (IV 27) aus dem Munde des Acacius selbst hat, usw.

\section{Theodoret als Geschichtschreiber}

Theodorets ,Art, Geschichte zu schreiben, wenn man diese Bezeichnung bei einem Autor gebrauchen darf, der sich zur Aufgabe gemacht hatte, eine Apologie zu verfassen, hat wenig gemein mit der des Socrates, der als ehrlicher und maßvoller Laie die Ereignisse nach den Gesetzen der Historiographie erzählen wollte (VI Prooem. 9), der sich weigerte, die

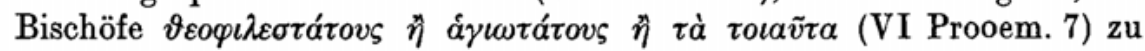
nennen, und der sogar scharfe Pfeile auf sie abzuschießen wagte (I 24,1)“.

Im Jahre 448 erhielt Theodoret wegen seines Eintretens für Nestorius vom Kaiser Theodosius II. den Befehl, das Gebiet seiner Diözese nicht zu verlassen. Die Reise zum Konzil von Ephesus August 449 wurde ihm untersagt, und auf dieser sog. Räubersynode wurde er abgesetzt. Er richtete daraufhin ein Schreiben an Papst Leo den Großen (Epist. 113) und berief sich darin, um seine Rechtgläubigkeit darzutun, auf seine bisherigen Schriften. Die KG wird unter diesen nicht erwähnt, existierte also noch 
nicht. Andererseits erzählt Theodoret am Schluß seiner KG $(338,14)$,

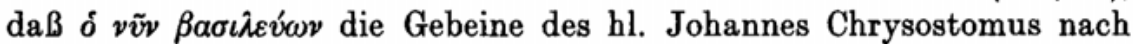
Konstantinopel habe zurückbringen lassen. ' $O$ vข̃v $\beta \alpha \sigma \iota \lambda \varepsilon v ́ \omega v$ aber ist Theodosius II., der am 28. Juli 450 starb. Theodoret hat demnach die KG in den letzten Monaten 449 und der ersten Hälfte 450 abgefaßt, als er wieder im Kloster Nicertae bei Apamea, wo er vor seiner Berufung zum Bischof als Mönch gelebt hatte, weilte und über die nötige Muße verfügte.

Er hat also kaum ein Jahr Zeit gehabt, um seine KG zu schreiben. „Stil und Abfassung sind weniger sorgfältig als in der Religiosa historia und in der Graecarum affectionum curatio. Theodoret hat sich begnügt, massenweise Urkunden abschreiben zu lassen, die er zur Verfügung hatte, für das übrige schöpfte er aus seinen andern Quellen und besonders aus seiner eignen umfangreichen Kenntnis, ohne sich um die Genauigkeit und um die Chronologie viel zu kümmern, indem er seine Sätze immer in derselben geistlichen Beredsamkeit bildete und diktierte, die ihm seit lange vertraut war."

„Die Erzählung hört mit 428 auf, dem Todesjahr des Theodorus von Mopsuestia und des Theodotus von Antiochien. Dies ist aber zugleich das Jahr, wo Nestorius zum Bischof von Konstantinopel erhoben wurde. Theodoret hätte die Geschichte der Kirche, in der er selber bald eine wichtige Rolle spielen sollte, nicht weiterführen können, ohne seinen eigenen Namen hineinzubringen."

„Theodoret hat jedenfalls aufrichtig geglaubt, daß es eine echte Geschichte der Vergangenheit seiner Kirche war, was er schrieb." Eine solche zu schreiben aber ist ihm nicht gelungen.

„Einige Beispiele lehren rasch erkennen, daß das Theodoret eigene Verfahren nicht der wahren Geschichtschreibung, sondern der kirchlichen Apologie angehört."

„Der Apologet muß seine Gegner - das sind in diesem Fall die Ketzer - ins Schwarze malen. Ein Bischof, der zugleich wie Theodoret Mönch ist, handelt in gutem Glauben, wenn er alle Ketzer für elende Betrüger hält. Von der ersten Seite an wird er für den Ursprung des Arianismus die Erklärung wählen, die nur der stärksten Glaubenseinfalt einleuchten mag. Der Priester Arius, ein Werkzeug des Teufels, handelt bloß aus Neid gegen seinen Erzbischof Alexander und beginnt, da er keinen Vorwand finden kann, dessen Leben zu verleumden, seine Lehre anzugreifen (S. 6, 14ff.). Dem Übel, das er anrichtet, $\mathrm{muB}$ schnell das schreckliche Ende eines solchen Ungeheuers gegenübergestellt werden. Aus diesem Grunde erzählt Theodoret aller Chronologie zum Trotz den Tod des Arius unmittelbar nach dem Konzil von Nicaea (I 14).“ 
„Niemals vergißt er, ähnliche Todesfälle von Gottlosen hervorzuheben (vgl. S. 183, $6 \mathrm{ff}$. III 13), selbst da, wo za fürchten ist, daß eine Verwechslung der Tatsachen vorliegt, z. B. 98, $6 \mathrm{ff}$. zwischen den Bischöfen Gregorius und Georgius von Alexandrien."

„Alle Ketzer werden als Verbrecher geschildert: Stephanus von Antiochien (II 9), Georgius und Lucius von Alexandrien (II 14; IV 21), Eudoxius (II 25 u. 27), Aëtius, Eunomius (II 27 u. 29) und sehr viele andere wie Leontius von Antiochien (II $10 \mathrm{u}$. 24), dessen sanfter Charakter bei orthodoxen Schriftstellern wie Sozomenus (III 20, 8-9) Gnade gefunden hatte. Die Predigt des Evangeliums bei den Goten ist eine Ruhmestat der orientalischen Kirche. Aber die Goten sind Arianer: einfach weil Eudoxius den Ulfilas bestochen hat $(274,6$ f.)."

„Und nun gar erst die nicht-orthodoxen Kaiser! Constantius ist ein $\varepsilon v ้ \varrho ı \pi \circ \varsigma$, den seine Höflinge nach allen beliebigen Winden drängen. Julian hat den Athanasius nicht nur aus Alexandrien verjagt, sondern er hat auch den Befehl gegeben, ihn zu töten (S. 186, 1). Es ist eine feststehende Tatsache, daß beim Drohen des Gotenkrieges Valens klugerweise alle Kräfte des Reiches zusammenfassen wollte und die rechtgläubige Geistlichkeit aus der Verbannung zurückberief (Hieronym. Chron. a. Abr. 2394; Rufin XI 13; vgl. Sozomen. VI 37, 1; Socrat. IV 37). Nach Theodoret dagegen setzte er seinenKampf gegen die göttlicheWahrheit fort (S. 271,2). Die Schlacht bei Adrianopel, in der Valens umkam, war ein furchtbares Unglück für das römische Heer. Theodoret erblickt darin aber nichts anderes als eine Züchtigung des Kaisers durch die göttliche Gerechtigkeit (IV 36).“

„Ist so auf der einen Seite kräftiger Haß gegen die Ketzer für Theodoret Pflicht, so kommt es ihm, dem Apologeten, anderseits nicht zu, die Fehler der Orthodoxen aufzudecken. Christliche Liebe und Furcht vor dem öffentliçhen Ärgernis befehlen ihm, sie zu verbergen. Das erhebt er sogar zum Grundsatz. In dem Augenblick, wo er die Leiden des Chrysostomus erzählt, erklärt er, daß er die Namen seiner Gegner verschweigen werde

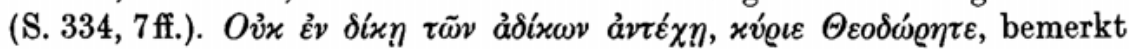
dazu ein ehrliches Scholion des alten Codex Vaticanus V. Diese ganze Geschichte des Chrysostomus, die Theodoret besser als jemand sonst kannte (Photius Biblioth. Cod. 273 las noch fünf Reden von ihm über den großen Patriarchen), erzählt er auf zwei Seiten nur ganz obenhin, und zwar aus dem Grunde, weil sich unter den Anstiftern zur Verfolgung des Chrysostomus neben dem Ägypter an erster Stelle seine Freunde befanden, der große Acacius von Beröa, Porphyrius von Antiochien und

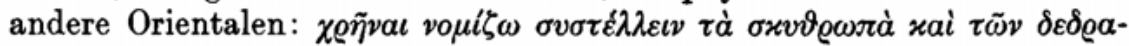

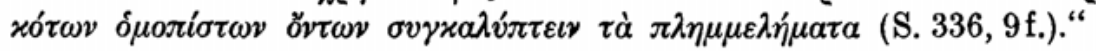


„Für solche Grundsätze beruft sich Theodoret auf die Autorität des großen Constantin. Bekannt ist die Geschichte der Anklageschriften gegen gewisse Bischöfe, die dem Kaiser während des Konzils von Nicaea überreicht wurden und die er ungelesen verbrennen ließ. Bei Socrates (I 8, 18-19), Sozomenus (I 17, 3-5), Rufinus (X 2) sind die Ankläger Bischöfe. Für Theodoret (S. 47, 9ff.) werden daraus einfach böswillige Menschen, und nun läßt er, indem er die ganze Tendenz der Geschichte ändert, Constantin sagen: ,Der Menge dürfen die Fehler der Bischöfe nicht enthüllt werden, weil zu befürchten steht, daß, wenn sie dort einen Anlaß zum Ärgernis findet, sie sich danach für berechtigt hält, selbst zu sündigen.” Und der Kaiser soll sogar hinzugefügt haben, er würde, wenn er mit eigenen Augen sähe, wie ein Bischof Ehebruch beginge, das Verbrechen mit seinem Purpur zudecken, damit ein solcher Anblick nur keinem Augenzeugen Schaden brächte."

Sonach darf Theodoret ,kaum als Autorität gelten, wenn Grund zu dem Verdacht vorliegt, daß seine Darstellung im Hinblick auf die gute Sache gefärbt sei. Als z. B. auf dem Konzil zu Mailand (355) die Bischöfe von Constantius angehalten wurden, zwischen der Verbannung und der Unterzeichnung von Athanasius' Verurteilung zu wählen, entschieden sich nur einige für die Verbannung, die andern bequemten sich schließlich zu dem notwendigen Opfer (Sozomen. IV 9, 1-4). Nach Theodoret (II 15) wollte kein Bischof unterzeichnen, sondern alle entschieden sich für die Verbannung."

,Dementsprechend darf Constantin, das Ideal eines christlichen Kaisers, nie wirklich schuldig gewesen sein. Wenn er Athanasius in die Verbannung geschickt hat, ist es entschuldbar, da $\beta$ er sich einmal hat täuschen lassen, wie das ja auch dem heiligen König und Propheten David widerfuhr (I 33). Man erwarte nicht, in Theodorets Buche eine Erwähnung der auf Constantins Befehl auf der Synode von Jerusalem erfolgten Restitution des Arius zu finden.... Wenn wir nur Theodoret hätten, würden wir glauben, daß der Umschwung zugunsten des Arianismus erst unter Constantius begonnen habe; wir hätten keine Kenntnis davon, daß Constantin durch den Arianer Eusebius von Nicomedien getauft worden ist, und wir wüßten nur, daß er auf seinem Sterbebett Athanasius, jenem Eusebius zum Trotz, zurückberufen hat (I 32), eine Zurückberufung, die im Grunde bloß durch die verdächtige Angabe Constantins II. in dem Brief an die Gemeinde von Alexandrien verbürgt ist (II 2)."

,Der große Theodosius ist eine Art neuer Constantin, auf den Theodoret die Motive ïberträgt, die schon bei jenem Verwendung gefunden hatten. Man vergleiche die Kundgebungen Constantins gegenüber den Bischöfen von Nicaea mit denen des Theodosius gegenüber Meletius auf 
dem Konzil zu Konstantinopel. Es geht das bis zur Wiederholung der-

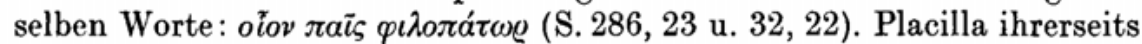
ist eine zweite Helena, und ihre Lobpreisung (V 19) ist nur eine Neuauflage von der auf die Mutter Constantins (I 18, S. 65, 10ff.).“

„Diese ganze Ökonomie von Theodorets Erzählung hat nur den einen Zweck: Verherrlichung der wahren Kirche und Erhöhung ihrer Diener. Groß ist der Bischof, der sich der Rechte der Kirche bewußt ist und der

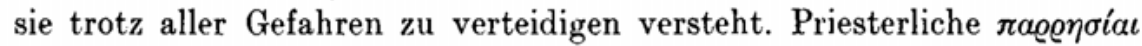
bilden ein immer wiederkehrendes Thema für die hochtönende Naivität Theodorets. Groß ist ein Kaiser, der in vollen Umfang die Rechte der Bischöfe anzuerkennen versteht und sich von ihnen Weisungen geben läßt."

„Als Ambrosius einige Tage nach seiner Wahl Valentinian I. gewisse Fehler seiner Beamten vorwirft (S. 219, 12ff.; vgl. Rufin. H. E. XI 11; Georg. Mon. 559, 5), läßt allein Theodoret den Kaiser antworten: ,Ich

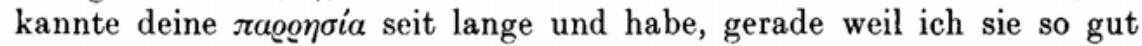
kannte, deine Wahl gewünscht." Es ist besser, in der Achtung vor den Geistlichen die Gewissenhaftigkeit zu weit zu treiben, als sich der Gefahr des Gegenteils auszusetzen. Man lese in dieser Hinsicht das merkwürdige Geschichtchen, das von Theodosius II. berichtet wird V 37, S. 339, 9 ff. Vor allem ist der große Theodosius unter diesem Gesichtspunkt das Muster eines Kaisers: $\imath \delta \iota \alpha$ (S. 309, 21f.). Gerade bei ihm hat die freie Erfindung Theodorets in der Umgestaltung der Geschichte des Ambrosius ihr Meisterwerk vollbracht. ... In der Auffassung Theodorets scheint das Blutbad von Thessalonich nur stattgefunden $\mathrm{zu}$ haben, um die Haupttugenden des

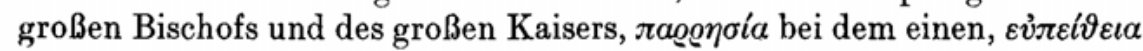
bei dem andern (S. 313, 7 ff.), glänzen zu lassen." 



\section{THEODORET}

\section{KIRCHENGESCHICHTE}

